\title{
Linear analysis of MESFET injection-locked oscillator array
}

\author{
C.-C. Hsiao and T.-H. Chu
}

\begin{abstract}
Quantitative expressions on the locking performance of a MESFET injection-locked oscillator (ILO) array are presented using nodal-admittance matrix representation. The formulations are derived on a linear approach, hence they are effective in the ILO array design. The locking performance of the ILO array includes the free-running frequency, output power and locking bandwidth. The derived formulations are verified using the measured results from a MESFET ILO and a four-element ring-type coupled ILO array.
\end{abstract}

\section{Introduction}

Stabilised oscillators are important in microwave electronic circuits. Injection locking of microwave solid-state oscillators is an effective approach to stabilise the oscillation frequency and improve the noise performance. In addition, the injection-locking approach can be found in a variety of applications, such as power combining, beam steering, amplifying, frequency dividing/multiplying and signal modulation [1-13]. It is then useful and necessary to derive effective formulations for the design of an injection-locked oscillator (ILO) array.

The injection-locking phenomenon has been widely discussed in the literature [1-26]. Adler [14] was one of the first to study the electronic-locking phenomenon and developed an expression for the frequency range over which an oscillator will remain locked in phase. He also developed a differential equation describing the oscillator phase as a function of time, which yields an understanding of the basic locking phenomena with the use of a simple physical model. Adler's work provided the foundation for the subsequent papers by Kurokawa [15], Stephan [17] and York et al. $[18,20,22]$. Kurokawa analysed the injection-locking phenomenon of a two-terminal negative-resistance oscillator using a graphical approach in terms of device and circuit admittance loci. Stephan and York then extended the existing theory to describe the phase and amplitude dynamics of coupled oscillators. To extend the analysis to the case of the two-port oscillator network, nonlinear methods such as the Volterra series method, the power series method or the harmonic balance method are employed to analyse the locking performance of various injection-locking arrangements $[9,19,20]$. However, the reported results in the literature do not give a straightforward formulation and the insight into the influence of device characteristics on the locking performance of ILO arrays.

(C) The Institution of Engineering and Technology 2006

IEE Proceedings online no. 20050027

doi:10.1049/ip-map:20050027

Paper first received 4th February 2005 and in revised form 19th April 2006

The authors are with the Graduate Institute of Communication Engineering, National Taiwan University, No. 1, Sec. 4, Roosevelt Road, Taipei, Taiwan 106, Republic of China

E-mail: thc@ew.ee.ntu.edu.tw
In this paper, we present the formulations for analysing the locking performance of an MESFET ILO array, using a linear approach based on the nodal-admittance matrix representation. In this approach, the modified linear expressions for the nonlinear elements $g_{m}, g_{d s}$ and $C_{g s}$ in a MESFET equivalent circuit are used. This is then an effective approach to derive the linear equations for the ILO-array locking-performance analysis. From the results, the locking performance is not only expressed in an explicit form, but also given as physical meaning.

\section{MESFET ILO array analysis}

\subsection{ILO array formulation}

As shown in Fig. 1 of an $N$-element ILO array, each ILO has an injection signal $i_{i n j, i}$. The mutual coupling among the oscillators at the output loads then generates additional injection signals which will affect the ILO-array locking performance. To quantitatively analyse this mutual coupling effect, the coupled signals of the $i$ th ILO from other oscillators are modelled by an equivalent current source $I_{c p, i}$, as shown in Fig. 2. In the following analysis, a ringtype nearest-neighbour coupling, as shown in Fig. 1, is employed for simplicity, therefore the coupling current $I_{c p, i}$ in Fig. 2 can be approximated as

$$
I_{c p, i} \approx Y_{i, i-1}\left(V_{2, i-1}-V_{2, i}\right)+Y_{i, i+1}\left(V_{2, i+1}-V_{2, i}\right)
$$

where $i=1,2, \ldots, N . V_{2, i}$ is the output voltage of the $i$ th oscillator, $V_{2,0}=V_{2, N}$ and $V_{2, N+1}=V_{2,1} . Y_{i, j}$ is the

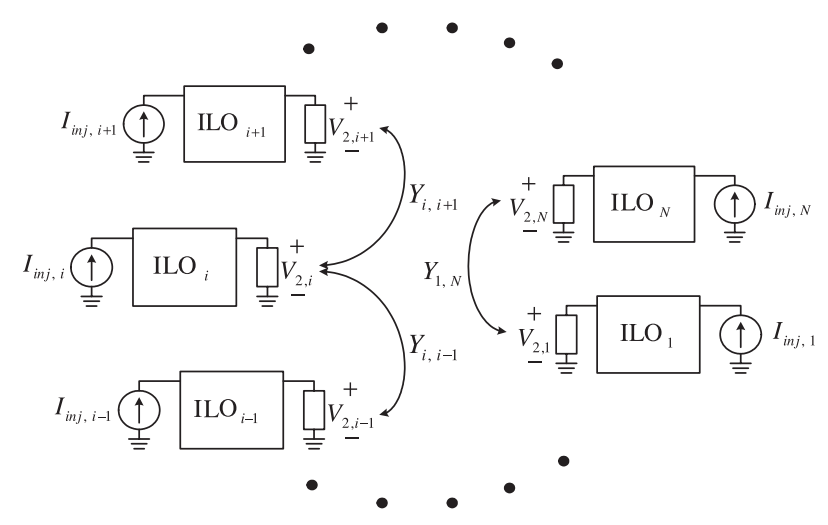

Fig. 1 Schematic diagram of a ring-type nearest-neighbour coupling $N$-element ILO array 


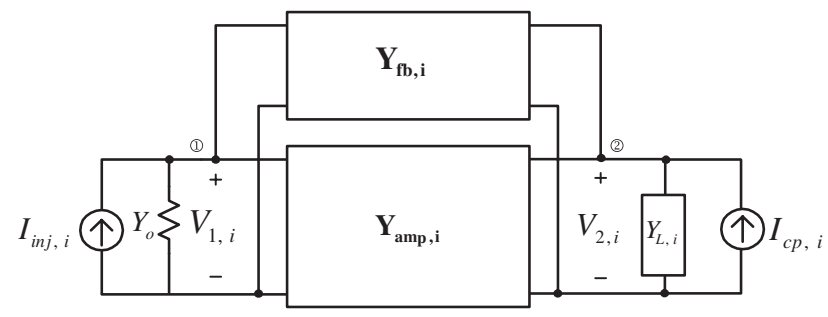

Fig. 2 Block diagram of the ith oscillator

admittance parameter between the $i$ th and the $j$-th ports of the coupling network.

From Fig. 2, the KCL equations of the ith ILO then give

$$
\boldsymbol{Y}_{i}(\omega)\left[\begin{array}{l}
V_{1, i} \\
V_{2, i}
\end{array}\right]=\left[\begin{array}{l}
I_{i n j, i} \\
I_{c p, i}
\end{array}\right]
$$

where

$$
\begin{aligned}
\boldsymbol{Y}_{i}= & \boldsymbol{Y}_{f b, i}+\boldsymbol{Y}_{a m p, i} \\
& +\left[\begin{array}{cc}
Y_{o} & 0 \\
0 & Y_{L, i}
\end{array}\right] \triangleq\left[\begin{array}{cc}
y_{11, i}+Y_{o} & y_{12, i} \\
y_{21, i} & y_{22, i}+Y_{L, i}
\end{array}\right]
\end{aligned}
$$

is the nodal admittance matrix of the $i$ th oscillator. $Y_{o}$ and $Y_{L, i}$ are the source and load admittances of the $i$ th ILO.

Considering the case of small-signal injection, (2) can be reduced to

$$
V_{2, i}=\frac{1}{\Delta_{i}}\left[-y_{21, i} I_{i n j, i}+\left(Y_{o}+y_{11, i}\right) I_{c p, i}\right]
$$

where $\Delta_{i}=\operatorname{det}\left(\boldsymbol{Y}_{i}\right)=\left(y_{11, i}+Y_{o}\right)\left(y_{22, i}+Y_{L, i}\right)-y_{12, i} y_{21, i}$. For simplicity, the admittance parameters of this coupling network are assumed to be identical with $Y_{i, j}=m$, for $|i-j|=1$. Thus, (1) becomes

$$
I_{c p, i} \approx m\left(V_{2, i-1}+V_{2, i+1}-2 V_{2, i}\right)
$$

where $m$ can be regarded as the mutual coupling coefficient of the ILO array.

By substituting (4) into (3), an $N$-oscillator array coupled through a ring-type nearest-neighbour coupling network can be expressed in a matrix form as

$$
\boldsymbol{M V}=\boldsymbol{y}_{21} \boldsymbol{U} \boldsymbol{I}_{i n j}
$$

where $\boldsymbol{M}$ is an $N \times N$ matrix with elements $M_{i j}$ :

$$
M_{i j}= \begin{cases}\Delta_{i}+2 m\left(Y_{o}+y_{11, i}\right) & i=j \\ -m\left(Y_{o}+y_{11, i}\right) & |i-j|=1 \\ 0 & \text { otherwise }\end{cases}
$$

In $(5), \boldsymbol{V}=\left[V_{2,1}, V_{2,2}, \ldots, V_{2, N}\right]^{T}, \boldsymbol{y}_{21}=\left[y_{21,1}, y_{21,2}, \ldots\right.$, $\left.y_{21, N}\right]^{T}, \boldsymbol{I}_{i n j}=\left[I_{i n j, 1}, \mathbf{I}_{i n j, 2}, \ldots, I_{i n j, N}\right]^{T}$ and $\boldsymbol{U}$ is an $N \times N$ identity matrix.

As the ILO array is in free-running mode, (5) becomes

$$
\boldsymbol{M V}=\mathbf{0}
$$

The frequency and amplitudes of the resulting oscillation are then solved from (7) subject to $\operatorname{det}(\boldsymbol{M})=0$.

To obtain the formulation for the maximum locking bandwidth of an ILO array, one can rewrite (3) as

$$
\begin{aligned}
V_{2, i}\left[\left(y_{11, i}+Y_{o}\right)\left(y_{22, i}+Y_{L, i}\right)-y_{12, i} y_{21, i}\right]= & -y_{21, i} I_{i n j, i} \\
& +\left(Y_{o}+y_{11, i}\right) I_{c p, i}
\end{aligned}
$$

or

$$
\begin{aligned}
y_{12, i} y_{21, i} V_{2, i}= & \left(y_{11, i}+Y_{o}\right)\left(y_{22, i}+Y_{L, i}\right) V_{2, i}+y_{21, i} I_{i n j, i} \\
& -\left(Y_{o}+y_{11, i}\right) I_{c p, i}
\end{aligned}
$$

to give

$$
\begin{aligned}
V_{2, i}= & \frac{\left(Y_{o}+y_{11, i}\right)\left(Y_{L, i}+y_{22, i}+2 m\right)}{y_{12, i} y_{21, i}} V_{2, i}+\frac{1}{y_{12, i}} I_{i n j, i} \\
& -\frac{Y_{o}+y_{11, i}}{y_{21,} y_{12, i}} m\left(V_{2, i+1}+V_{2, i-1}\right) \\
= & \frac{\left(Y_{o}+y_{11, i}\right)\left(Y_{L, i}+y_{22, i}+2 m\right)}{y_{12, i} y_{21, i}} V_{2, i}+\frac{1}{y_{12, i}} I_{i n j, i} \\
& \times\left[1-\frac{Y_{o}+y_{11, i}}{y_{21, i}} \frac{m\left(V_{2, i+1}+V_{2, i-1}\right)}{I_{i n j, i}}\right] \\
= & \frac{\left(Y_{o}+y_{11, i}\right)\left(Y_{L, i}+y_{22, i}+2 m\right)}{y_{12, i} y_{21, i}} V_{2, i}+\frac{1}{y_{12, i}} I_{e q, i}
\end{aligned}
$$

where

$$
I_{e q, i}=I_{i n j, i}\left[1-\frac{Y_{o}+y_{11, i}}{y_{21, i}} \frac{m\left(V_{2, i+1}+V_{2, i-1}\right)}{I_{i n j, i}}\right]
$$

is the equivalent injection signal of the $i$ th oscillator.

For the case of small-signal injection locking and loose coupling, one can obtain the injection-locking bandwidth from the phase relationships between $V_{2, i},\left(1 / y_{12, i}\right) I_{e q, i}$, and

$$
\frac{\left(Y_{o}+y_{11, i}\right)\left(Y_{L, i}+y_{22, i}+2 m\right)}{y_{12, i} y_{21, i}} V_{2, i}
$$

as in [2]. That is,

$$
\Delta \omega_{\max }=\frac{2}{K_{i}\left|y_{12, i}\right|} \frac{\left|I_{e q, i}\right|}{\left|V_{2, i}\right|}
$$

where

$$
K_{i}=\frac{\mathrm{d}}{\mathrm{d} \omega}\left(\angle \frac{\left(Y_{o}+y_{11, i}\right)\left(Y_{L, i}+y_{22, i}+2 m\right)}{y_{12, i} y_{21, i}}\right)
$$

In the following, the locking performance analysis is applied to the case of a four-element MESFET ILO array.

\subsection{Modified MESFET linear model}

To quantitatively analyse the effect of a nonlinear MESFET on the injection locking behaviour, the modified linear expressions for the MESFET model are given as follows, based on the treatment given in [23]. Figure 3 illustrates the nonlinear equivalent circuit of a MESFET. Among the equivalent circuit elements, transconductance $g_{m}$, gatesource capacitance $C_{g s}$ and the output conductance $g_{d s}$ are bias-dependent nonlinear elements and can be approximated by the third-order power series expressions in the operation range near the bias point [23]. For an ILO, the fundamental mode is the dominant mode of operation. For this reason, as the ILO reaches its steady state, one can define the modified linear expression of $g_{m}, C_{g s}$ and $g_{d s}$ as [23]:

$$
\overline{g_{m}} \equiv g_{m 1}+\frac{3}{4} g_{m 3}\left|V_{g s}\right|^{2}
$$

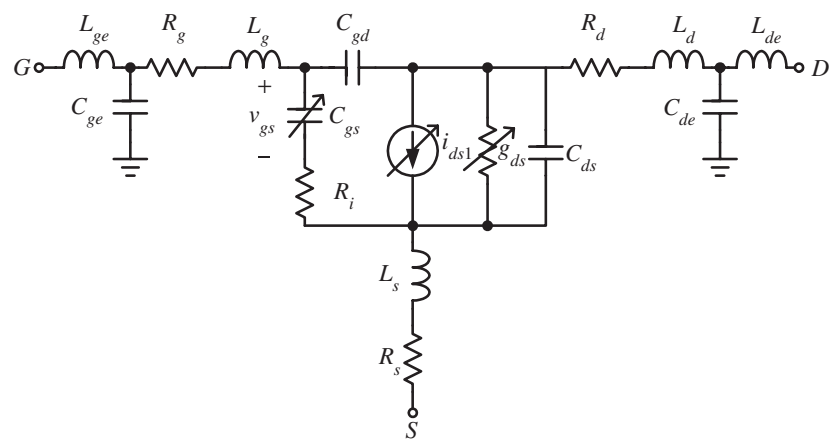

Fig. 3 Equivalent circuit of MESFET 


$$
\begin{gathered}
\overline{C_{g s}} \equiv C_{g s 1}+\frac{3}{4} C_{g s 3}\left|V_{g s}\right|^{2} \\
\overline{g_{d s}} \equiv g_{d s 1}+\frac{3}{4} g_{d s 3}\left|V_{d s}\right|^{2}
\end{gathered}
$$

In (12)-(14), $g_{m 1}, C_{g s 1}, g_{d s 1}$ and $g_{m 3}, C_{g s 3}, g_{d s 3}$ are the firstorder and third-order nonlinear coefficients of $g_{m}, C_{g s}$ and $g_{d s}$, respectively.

Accordingly, the MESFET can be regarded as a linear circuit, as shown in Fig. 4, with $\overline{g_{m}}, \overline{C_{g s}}$ and $\overline{g_{d s}}$ obtained from (12)-(14). This linearisation of the MESFET model simplifies the calculation of the nodal admittance matrix $Y_{i}$, therefore both $K_{i}$ and $y_{12, i}$ in (11) can be directly calculated.

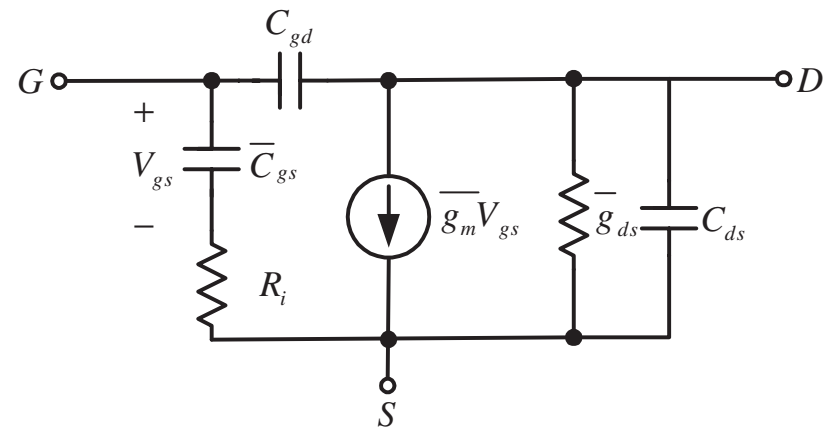

Fig. 4 Simplified linear model of MESFET

\subsection{Coupling in the ILO array}

Figure 5 shows the circuit of a four-element ring-type coupled MESFET oscillator array. The mutual coupling coefficient $m$ in (11) is given as [20]:

$$
m=-\frac{\mathrm{e}^{-\mathrm{j} \Phi}}{2 R} \Lambda
$$

where

$$
\Lambda=\frac{\cos \psi+\mathrm{j} \frac{R Z_{o}}{Z_{x}\left(R+Z_{o}\right)} \sin \psi}{\cos \psi+\mathrm{j} \frac{Z_{x}\left(R+Z_{o}\right)}{R Z_{o}} \sin \psi}
$$

and $\Phi$ and $\psi$ are the phase-delay terms of the transmission lines with impedances $R$ and $Z_{x}$, respectively. By substituting (15) into (7) and (11), the free-running frequency and the maximum locking range of an ILO array can then be calculated, respectively.

\section{Experimental results}

To verify the derived injection-locking bandwidth, a MESFET ILO and a four-element oscillator array are fabricated on a 0.6-mm thick FR4 substrate with $\varepsilon_{r}=4.3$. Figure 6 shows the ILO microstrip circuit. The MESFET NE32684A is biased at $V_{d s}=3 \mathrm{~V}$ and $I_{d s}=30 \mathrm{~mA}$. The simulation result of the oscillation frequency is $4.33 \mathrm{GHz}$ with 8-dBm output power, whereas the measured results are 7.5-dBm output power and an oscillation frequency of $4.34 \mathrm{GHz}$.

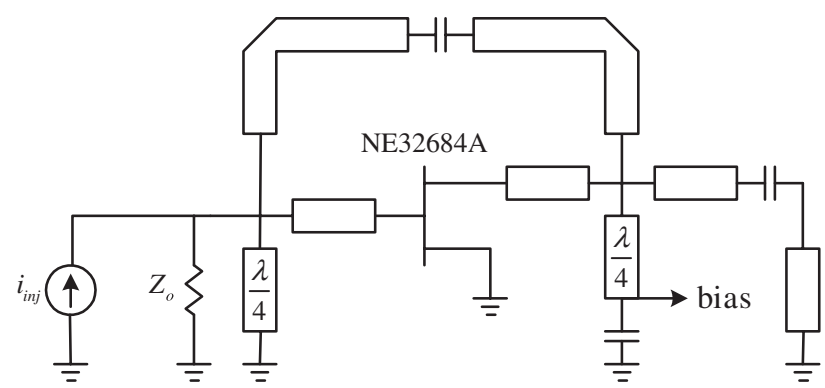

Fig. 6 ILO microstrip circuit

The calculated and measured locking ranges are shown in Fig. 7, which indicates that the locking bandwidth increases with the injection signal level. Note the locking bandwidth is normalised to the oscillator free-running frequency.

The circuit of a four-element ring-type coupled oscillator array, shown in Fig. 5, is designed to have an oscillation frequency at $4.27 \mathrm{GHz}$. The array coupling phase $\Phi=2 \pi$ is implemented by a $50-\Omega$ microstrip transmission line, loaded with two $50-\Omega$ chip resistors. In measuring the ILO array performance, all the four oscillators are tuned to have nearly identical free-running frequency. Figure 8 shows the experimental arrangement, with an HP83620A signal

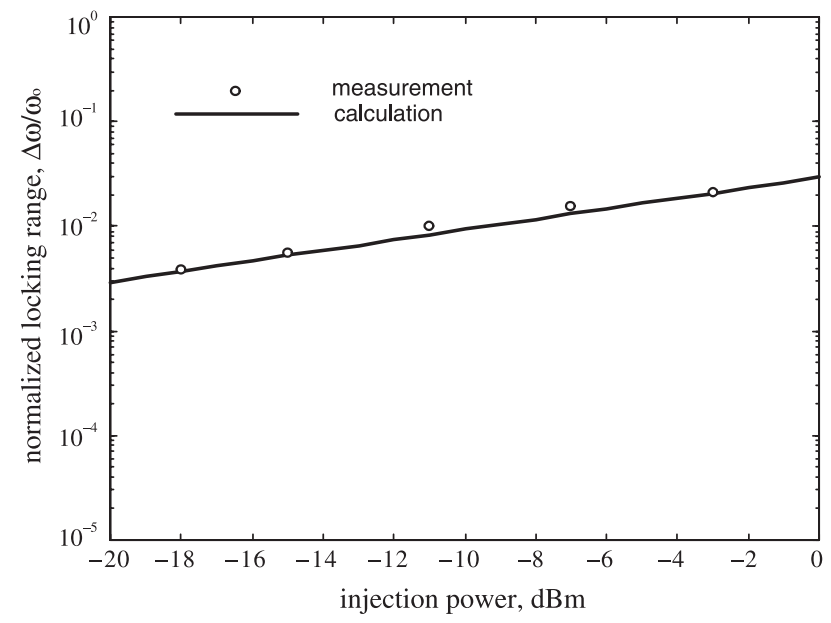

Fig. 7 Measured and calculated results of ILO locking bandwidth

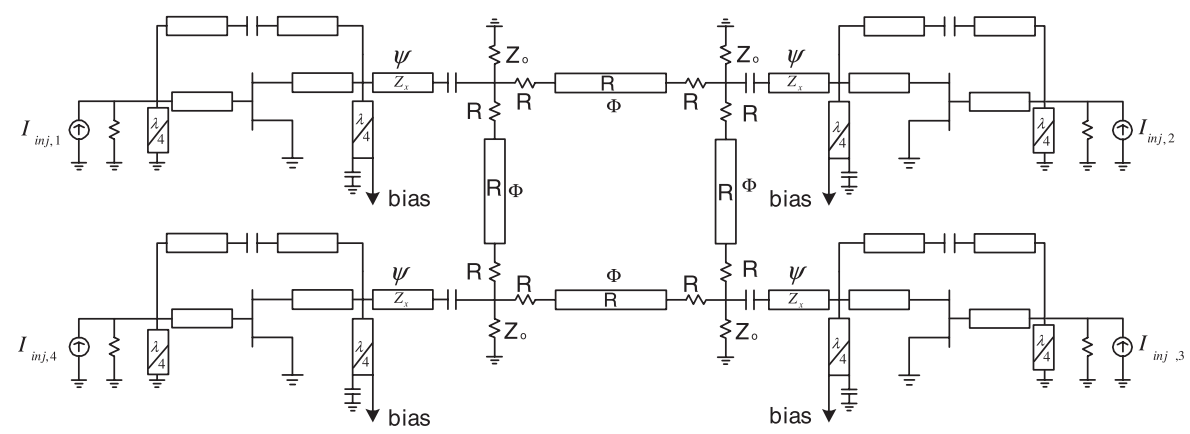

Fig. 5 4-element ring-type coupled oscillator array 


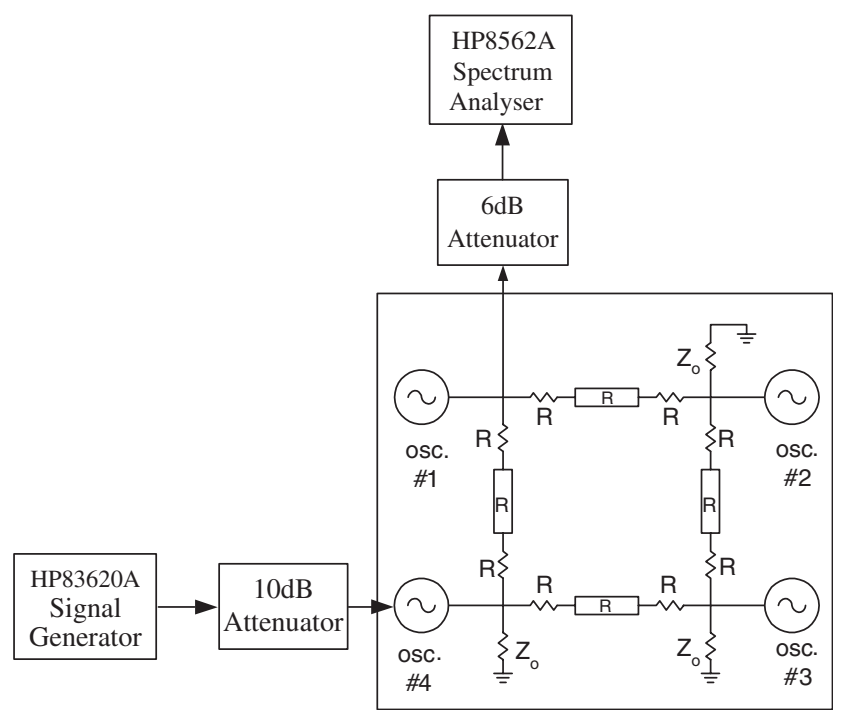

Fig. 8 Measurement arrangement of the locking range for the 4-element ring-coupled oscillator array

generator as the injection source and an HP8562A spectrum analyser to measure the output spectra. Two attenuators are used to improve the impedance mismatch of the injection source and spectrum analyser. Here, the injection signal is impressed only on one oscillator, and for convenience the locking range is measured at the output port of the other oscillator. The measured and calculated results of the injection-locking bandwidth are shown in Fig. 9, and are in good agreement.

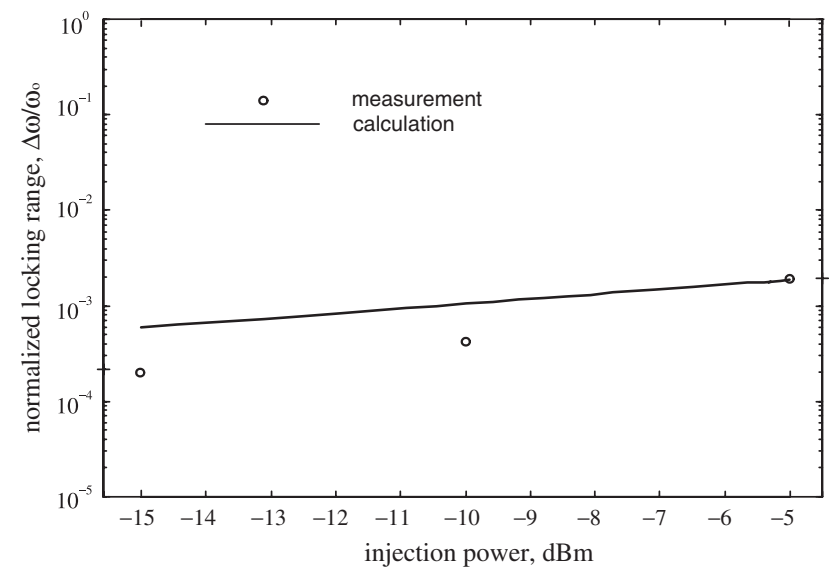

Fig. 9 Measured and calculated results of the locking bandwidth of a 4-element ring-type coupled oscillator array

\section{Conclusions}

A new method to calculate the locking range of the ILO array is presented. In the formulation of the ILO array, the mutual coupling is modelled as an equivalent current source and the nonlinear MESFET parameters are represented by modified linear expressions of $g_{m}, C_{g s}$ and $g_{d s}$. The derived equation is then useful in the ILOarray design. Both the analytical results of an ILO and an ILO array are verified with experimental measurements and shown to be in good agreement with the calculated results.

\section{Acknowledgments}

This work was supported by the National Science Council of Taiwan, R.O.C. under grants NSC 94-2213-E-002-010 and NSC 94-2752-E-002-004-PAE.

\section{References}

1 Zarroug, A., Hall, P.S., and Cryan, M.: 'Active antenna phase control using subharmonic locking', Electron. Lett., 1955, 31, (11), pp. 842-843

2 Birkeland, J., and Itoh, T.: 'A 16 element quasi-optical FET oscillator power combining array with external injection locking', IEEE Trans. Microw. Theory Tech., 1992, 40, pp. 475-481

3 Kykkotis, C., Hall, P.S., and Ghafouri-Shiraz, H.: 'Performance of active antenna oscillator arrays under modulation for communication systems', IEE Proc.-Microw. Antennas Propag., 1998, 145, (4), pp. 313-320

4 Qian, Y., and Itoh, T.: 'Progress in active integrated antennas and their applications', IEEE Trans. Microw. Theory Tech., 1998, 46, pp. $1891-1900$

5 Stephan, K.D.: 'Inter-injection-locked oscillators for power combining and phased arrays', IEEE Trans. Microw. Theory Tech., 1986, 34, pp. $1017-1025$

6 York, R.A., and Compton, R.C.: 'Quasi-optical power combining using mutually synchronized oscillator arrays', IEEE Trans. Microw. Theory Tech., 1991, 39, pp. 1000-1009

7 Pogorzelski, R.J.: 'On the dynamics of two-dimensional array beam scanning via perimeter detuning of coupled oscillator arrays', IEEE Trans. Antennas Propag., 2001, 49, pp. 234-242

8 Thamos $\mathrm{Jr}$., and Branner, D.G.: 'Optimization of active microwave frequency multiplier performance utilizing harmonic terminating impedances', IEEE Trans. Microw. Theory Tech., 1996, 44, pp. 2617-2624

9 Rategh, H.R., and Lee, T.H.: 'Superharmonic injection-locked frequency dividers', IEEE J. Solid-State Circuits, 1999, 34, pp. 813-821

10 Liao, P., and York, R.A.: 'A new phase-shifterless beam-scanning technique using arrays of coupled oscillators', IEEE Trans. Microw. Theory Tech., 1993, 41, pp. 1810-1815

11 Isobe, T., and Tokida, M.: 'A new microwave amplifier for multichannel FM signals using a synchronized oscillator', IEEE J. Solid-State Circuits, 1969, 4, pp. 400-408

12 Sommer, D., and Gomes, N.J.: 'Wide-locking bandwidth optically injection-locked oscillators: S-parameter design and modulation effects', IEEE Trans. Microw. Theory Tech., 1995, 43, pp. 1424-1432

13 York, R.A., and Itoh, T.: 'Injection- and phase-locking techniques for beam control', IEEE Trans. Microw. Theory Tech., 1999, 46, pp. 1920-1929

14 Adler, R.: 'A study of locking phenomena in oscillators', Proc. IEEE 1973, 61, (10), pp. 1380-1385 (reprinted from proc. IRE, 1946, 34, pp. 351-357)

15 Kurokawa, K.: 'Injection locking of microwave solid-state oscillators', Proc. IEEE, 1973, 61, pp. 1386-1410

16 Uzunoglu, V., and White, M.H.: 'The synchronous oscillator: a synchronization and tracking network', IEEE J. Solid-State Circuits, 1985,20 , pp. $1214-1225$

17 Stephan, K.D., and Morgan, W.A.: 'Analysis of interinjection-locked oscillators for integrated phased arrays', IEEE Trans. Antennas Propag., 1987, 35, pp. 771-781

18 York, R.A.: 'Nonlinear analysis of phase relationships in quasi-optical oscillator arrays', IEEE Trans. Microw. Theory Tech., 1993, 41, pp. 1799-1809

19 Hwang, C.C., and Chu, T.H.: 'Analysis of MESFET injection-locked oscillators in fundamental mode of operation', IEEE Trans. Microw. Theory Tech., 1994, 42, pp. 1851-1857

20 York, R.A., Liao, P., and Lynch, J.J.: 'Oscillator array dynamics with broadband N-port coupling networks', IEEE Trans. Microw. Theory Tech., 1994, 42, pp. 2040-2045

21 Humphrey, D.E.J., Fusco, V.F., and Drew, S.: 'Active antenna array behavior', IEEE Trans. Microw. Theory Tech., 1995, 43, pp. 1819-1824

22 Chang, H.C., Shapiro, E.S., and York, R.A.: 'Influence of the oscillator equivalent circuit on the stable modes of parallel-coupled oscillators', IEEE Trans. Microw. Theory Tech., 1997, 45, pp. 12321239

23 Yang, Y.R., and Chu, T.H.: 'Locking performance analysis of MESFET subharmonically injection-locked oscillator', IEEE Trans. Microw. Theory Tech., 1999, 47, pp. 1014-1020

24 Chang, H.C., Borgioli, A., Yeh, P., and York, R.A.: 'Analysis of oscillators with external feedback loop for improved locking range and noise reduction', IEEE Trans. Microw. Theory Tech., 1999, 47, pp. $1535-1543$

25 Moyer, H.P. and Daryoush, A.S.: 'A unified analytical model and experimental validations of injection-locking processes', IEEE Trans. Microw. Theory Tech., 2000, 48, pp. 493-499

26 Randall, M., and Hock, T.: 'General oscillator characterization using linear open-loop S-parameters', IEEE Trans. Microw. Theory Tech. 2001, 49, pp. 1094-1100 